\title{
PICARD-LEFSCHETZ THEOREM FOR FAMILIES OF NONSINGULAR ALGEBRAIC VARIETIES ACQUIRING ORDINARY SINGULARITIES
}

\author{
BY \\ C. H. CLEMENS, JR.
}

1. Introduction. The purpose of this paper is to expand the classical results of Picard [6] and Lefschetz [3, p. 23] concerning a one-complex-parameter family of algebraic varieties $S_{t}$ acquiring singularities for isolated values of $t$. The question to be answered is: What action on $H_{*}\left(S_{t}\right)$ is induced by motion of the parameter $t$ ? $\left(H_{*}(\quad)\right.$ will denote (compact) singular homology with real coefficients.)

More precisely, let $B$ be a complex analytic line bundle over a nonsingular complex projective variety $X$ of (complex) dimension $(n+1)$. Let $s_{0}$ and $s_{\infty}$ be any two linearly independent holomorphic sections of $B \rightarrow X$ such that $Z\left(s_{\infty}\right) \cap Z\left(d s_{\infty}\right)$ $=\varnothing .\left(Z(\quad)\right.$ will indicate "zeros of $(\quad)$ in $X$.") Let $S_{t}=Z\left(s_{0}+t s_{\infty}\right)$ and $S_{\infty}=Z\left(s_{\infty}\right)$. Then $\left(s_{\infty} / s_{0}\right)$ is a meromorphic and hence algebraic function on $X$, and so $S_{t}$ is a projective hypersurface of $X$ for all $t$. Since $S_{\infty}$ is assumed nonsingular, standard arguments show that

$$
C_{0}=\left\{t \in C: Z\left(s_{0}+t s_{\infty}\right) \cap Z\left(d s_{0}+t d s_{\infty}\right) \neq \varnothing\right\}
$$

is finite.

For $t \in\left(C-C_{0}\right), S_{t}$ has a $C^{\infty}$-normal bundle $W$ in $X$ whose fibres are ordinary two-real-dimensional discs. Let the fibration be given by $\omega: W \rightarrow S_{t}$. For $t^{\prime}$ near $t$, $S_{t^{\prime}}$ is also nonsingular, and for each $P \in S_{t}$ the set $\left(\omega^{-1}(P) \cap S_{t^{\prime}}\right)$ contains exactly one point. Thus $\omega$ defines a diffeomorphism $F\left(t, t^{\prime}\right): S_{t^{\prime}} \rightarrow S_{t}$. Given any path $\gamma$ in $\left(\boldsymbol{C}-\boldsymbol{C}_{0}\right)$, beginning at $t_{0}$ and ending at $t$, we can define an associated diffeomorphism $F(\gamma)=F\left(t_{k}, t_{k-1}\right) \circ \cdots \circ F\left(t_{2}, t_{1}\right) \circ F\left(t_{1}, t_{0}\right)$ where $\left\{t_{0}, t_{1}, \ldots, t_{k}=t\right\}$ is a sufficiently fine partition of $\gamma$. The homotopy type of $F(\gamma)$ does not depend on the choice of normal bundles [4, pp. 19-26], nor on the partition if it is sufficiently fine, but only on the homotopy class of $\gamma$ in $\left(C-C_{0}\right)$.

One asks, then, what is the associated homology isomorphism $F(\gamma)_{*}: H_{*}\left(S_{t_{0}}\right)$ $\rightarrow H_{*}\left(S_{t}\right)$ ? From the discussion above, it clearly suffices to consider the case $0 \in C_{0},\{t: 0<|t|<2\} \subseteq\left(C-C_{0}\right)$, and $\gamma=\{\exp \xi: 0 \leqq \xi \leqq \theta\}$ where exp $\xi=e^{2 \pi i \xi}$. For such situations, define $F(\theta)=F(\gamma)$. In [3, p. 23], Lefschetz describes $F(q)_{*}: H_{*}\left(S_{1}\right)$ $\rightarrow H_{*}\left(S_{1}\right)$ for $q \in Z$, when $S_{0}$ has isolated double points as its singular locus. This result is variously called the Picard-Lefschetz Theorem or the Poincaré formula. Pham [5] extended these results to much more general types of isolated singularities. In the following we will generalize in another direction and allow $\left\{S_{t}\right\}$ to acquire an

Received by the editors February 14, 1966 and, in revised form, October 16, 1967. 
arbitrary ordinary singularity at $S_{0}$. Our general philosophy will be to fibre the action of $F(\theta)$ over the singular locus of $S_{0}$. (This approach also yields results in some simple cases of nonordinary, nonisolated singularities [1].) We will assume throughout that $\{0<|t|<2\} \subseteq\left(C-C_{0}\right)$.

Theorem 4.4 of this paper will give, from the topological point of view, an appropriate generalization of the classical Picard-Lefschetz Theorem to the case in which we acquire an arbitrary singularity (with arbitrary multiplicities) at $S_{0}$. A. Landman, in his Berkeley thesis, treated this same problem from an algebraic point of view. To see the relation between these two approaches, we might note here a corollary of the homological dimensions of the subspaces of $S_{1}$ in Theorem 4.4 and Corollary 4.15:

For $0 \leqq a \leqq 2 n$ and $m=$ least common multiple of the multiplicities of the components of $S_{0}$ and

$$
f_{a}=F(1)_{*}: H_{a}\left(S_{1}\right) \rightarrow H_{a}\left(S_{1}\right),
$$

the minimal polynomial of the linear transformation $f_{a}$ is a factor of the polynomial

$$
\left(\lambda^{m}-1\right)^{n+1-|a-n|} \text {. }
$$

2. Ordinary singularities and the fibration of $F(\theta)$. For notational convenience, we will set $S_{\infty}=A(0)$. Then

Definition 2.1. The family $\left\{S_{t}\right\}$ will be said to acquire an ordinary singularity at $t=0$ if:

(1) $S_{0}=A(1) \cup \cdots \cup A(h)$ where the $A(i)$ are nonsingular hypersurfaces of $X$;

(2) for $0 \leqq i<j \leqq h, A(i)$ and $A(j)$ intersect normally (including the possibility that $A(i) \cap A(j)=\varnothing)$.

From now on we assume that $\left\{S_{t}\right\}$ is as in $\S 1$ and acquires an ordinary singularity at $t=0$.

For any open set $U \subseteq X$, let $I(U)=\{i \in\{0, \ldots, h\}: U \cap A(i) \neq \varnothing\}$. Then locally we can normalize our defining equation $s_{0}+t s_{\infty}=0$ for $S_{t}$ :

LEMMA 2.2. There is an open covering $\{U\}$ of $S_{0} \cup S_{\infty}=A(0) \cup \cdots \cup A(h)$ in $X$ and complex analytic functions $x_{i}: U \rightarrow C$ for each $U \in\{U\}, i \in I(U)$ such that

(1) $\left(x_{i}\right)_{i \in I(U)}$ are part of an analytic coordinate system for $U$ in $X$;

(2) for each $U \in\{U\}, Z\left(x_{i}\right)=A(i) \cap U$;

(3) $-\left(\left(s_{0} / s_{\infty}\right)+t\right)=\left(\pi\left\{x_{i}^{m(i)}: i \in I(U)\right\}-t\right)$ on $U \in\{U\}$.

The $m(i)$ are integers whose values depend only on $i$ and not on the open set $U$. $m(0)=-1$, and $m(i)$ is a positive integer for $i>0$ and is called the multiplicity of $A(i)$ at $t=0$.

Proof. Theory of functions of several complex variables. See [2].

Definition 2.3. A finite open covering $\mathscr{V}$ of $S_{0} \cup S_{\infty}$ in $X$ will be called a regular covering if

(1) each $V \in \mathscr{V}$ is a simply connected subset of a coordinate disc in $X$;

(2) Lemma 2.2 holds for $\{U\}=\mathscr{V}$; 
(3) for $V \in \mathscr{V}, j \notin I(V), \bar{V} \cap A(j)=\varnothing$;

(4) for $V, V^{\prime} \in \mathscr{V}$ with $V \cap V^{\prime} \neq \varnothing, V \cap V^{\prime}$ is simply connected.

Next define $A(I)=\bigcap\{A(i): i \in I\}$ for $I \subseteq\{0, \ldots, h\}$ and

$$
B(q)=\bigcup\{A(I):|I|=q\} .
$$

THEOREM 2.4. There exists a regular covering $\mathscr{V}$ of $S_{0} \cup S_{\infty}=B(1)$ and $C^{\infty}$ mappings:

$$
\begin{gathered}
y(i, V):(A(I(V)) \cap V) \times V \rightarrow C \text { for } i \in I(V), \\
z(V):(A(I(V)) \cap V) \times V \rightarrow C^{(n+1-|I(V)|)}
\end{gathered}
$$

for each $V \in \mathscr{V}$ such that:

(1) $\left((y(i, V)(P, \quad))_{i \in I(V)}, z(V)(P, \quad)\right)$ forms a complex analytic coordinate system for $V$ centered at $P$ for each $P \in(A(I(V)) \cap V)$;

(2) on $V \in \mathscr{V},-\left(s_{0} / s_{\infty}+t\right)=\left(\pi\left\{y(i, V)(P, \quad)^{m(i)}: i \in I(V)\right\}-t\right)$;

(3) for $V \cap V^{\prime} \neq \varnothing$ and $I(V)=I\left(V^{\prime}\right)$ :

(a) $\left(y(i, V)\left(P_{0}, Q\right) / y\left(i, V^{\prime}\right)\left(P_{0}, Q\right)\right)$ is a constant with absolute value 1 for each fixed $P_{0} \in V \cap V^{\prime} \cap A(I(V)), i \in I(V)$,

(b) $Z\left(z(V)\left(P_{0}, Q\right)\right)=Z\left(z\left(V^{\prime}\right)\left(P_{0}, Q\right)\right)$ for each $P_{0}$;

(4) for $V \cap V^{\prime} \neq \varnothing$ and $I(V) \subseteq I\left(V^{\prime}\right)$ :

(a) for $i \in I(V),\left(y(i, V)\left(Q_{0}, Q\right) / y\left(i, V^{\prime}\right)\left(P_{0}, Q\right)\right)$ is a nonzero constant for $z(V)\left(Q_{0}, Q\right)=0$ for all $P_{0}, Q_{0}$ fixed such that $Q_{0} \in V \cap V^{\prime} \cap A(I(V)), P_{0} \in V^{\prime}$ $\cap A\left(I\left(V^{\prime}\right)\right)$, and $z\left(V^{\prime}\right)\left(P_{0}, Q_{0}\right)=0$,

$$
Z\left(z(V)\left(Q_{0}, Q\right)\right)=Z\left(z\left(V^{\prime}\right)\left(P_{0}, Q\right)\right)
$$

for all $P_{0}, Q_{0}$ as in (a);

$$
\cap \bigcap\left\{Z\left(y\left(i, V^{\prime}\right)\left(P_{0}, Q\right)-y\left(i, V^{\prime}\right)\left(P_{0}, Q_{0}\right)\right): i \in I\left(V^{\prime}\right)-I(V)\right\}
$$

(5) for all $V \in \mathscr{V}, V$ is the disjoint union of $Z(z(V)(P, Q))$ over $P \in(A(I(V)) \cap V)$.

Proof. Starting from $B(n+2)=\varnothing$, we shall assume that we have a finite cover $\mathscr{V}_{q+1}$ of $B(q+1)$ which satisfies (2.3), (1)-(4), and (2.4), (1)-(5), and shall construct a cover $\mathscr{V}_{q}$ with the corresponding properties over $B(q)$. Since $B(q)$ $-\bigcup\left\{V: V \in \mathscr{V}_{q+1}\right\}$ is a disjoint union of subspaces, one for each $I$ with $|I|=q$, it will clearly suffice to demonstrate the construction of the cover for $A(I) \cup B(q+1)$ for some $\dot{I}$ with $|I|=q$. Thus let $\mathscr{V}^{\prime}$ be a finite open covering of the compact set $A(I)-\bigcup\left\{V: V \in \mathscr{V}_{q+1}\right\}$ in $X$. By refining $\mathscr{V}_{q+1}$ if necessary we can assume that

(1) $I(V)=I$ for all $V \in \mathscr{V}^{\prime}$ and $|I(V)|>q$ for all $V \in \mathscr{V}_{q+1}$,

(2) $\mathscr{V}^{\prime} \cup \mathscr{V}_{q+1}$ satisfies (2.3), (1)-(4) as a finite open covering of $A(I) \cup B(q+1)$ in $X$. (For (2.3)(4), use a covering of $X$ by open stars with respect to a sufficiently fine triangulation.)

Let $\mathscr{V}^{\prime \prime}=\left\{V \in \mathscr{V}_{q+1}: I \subseteq I(V)\right\}$. For $V \in \mathscr{V}^{\prime}$, let $(x(i, V))_{i \in I}$ be the coordinates as in Lemma 2.2. Let $w(V): V \rightarrow C^{(n+1-q)}$ be such that $\left((x(i, V))_{I}, w(V)\right)$ form a set of coordinates for $V$ in $X$. Define $x(i, V)(P, Q)=x(i, V)(Q)$ for all $P \in A(I) \cap V$. Define $w(V)(P, Q)=w(V)(Q)-w(V)(P)$. 
For $V \in \mathscr{V}^{\prime \prime}$, define

$$
x(i, V)(P, Q)=y(i, V)\left(P_{0}, Q\right)
$$

where $P \in V \cap A(I)$ and $z(V)\left(P_{0}, P\right)=0$ and $i \in I$;

$$
w(V)(P, Q)=\left(z(V)\left(P_{0}, Q\right),\left(y(j, V)\left(P_{0}, Q\right)-y(j, V)\left(P_{0}, P\right)\right)_{j \in I(V)-I}\right)
$$

with $P, P_{0}$ as before.

Next for $V \in \mathscr{V}^{\prime}, V^{\prime} \in \mathscr{V}^{\prime} \cup \mathscr{V}^{\prime \prime}$ and $V \cap V^{\prime} \neq \varnothing$, define

$$
\begin{aligned}
& u\left(i, V, V^{\prime}\right)(P, Q) \\
& \quad=\left(x\left(i, V^{\prime}\right)(P, Q) \cdot \pi\left\{y\left(j, V^{\prime}\right)\left(P_{0}, Q\right)^{m(j) / q(m(i))}: j \in I\left(V^{\prime}\right)-I\right\}\right) / x(i, V)(P, Q)
\end{aligned}
$$

for $i \in I$ with $P \in A(I) \cap V \cap V^{\prime}, P_{0} \in A\left(I\left(V^{\prime}\right)\right) \cap V^{\prime}$ and $z\left(V^{\prime}\right)\left(P_{0}, P\right)=0$. Further, for $V, V^{\prime} \in \mathscr{V}^{\prime} \cup \mathscr{V}^{\prime \prime}$, let $\Delta\left(V, V^{\prime}\right)(P)=$ the Jacobian matrix

$$
\left.\left(\partial w(V)(P, Q) / \partial w\left(V^{\prime}\right)(P, Q)\right)\right|_{P} .
$$

Now if $\left\{\rho_{V}\right\}$ is a $C^{\infty}$ partition of unity of $A(I)$ subordinate $\left({ }^{1}\right)$ to the cover $\mathscr{V}^{\prime} \cup \mathscr{V}^{\prime \prime}$ define:

$$
y(i, V)(P, Q)=x(i, V)(P, Q) \cdot \pi\left\{u\left(i, V, V^{\prime \prime}\right)(P, Q) \rho_{V^{\prime \prime}}(P): V^{\prime \prime} \in \mathscr{V}^{\prime} \cup \mathscr{V}^{\prime \prime}\right\}
$$

for $V \in \mathscr{V}^{\prime}$ and $i \in I$. Further define

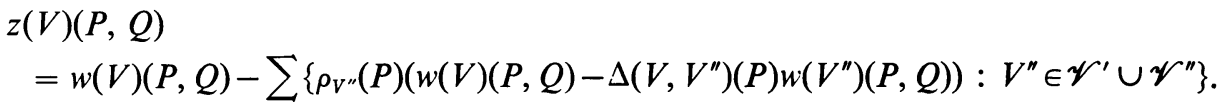

With appropriate restrictions of various open sets of the coverings, these mappings give the induction step and hence the theorem.

The above theorem leads us to consider what happens locally with respect to normalized coordinates.

3. The normalized case. For the present chapter only, let $S_{t}=Z\left(y_{1}^{m_{1}} \cdots \cdots y_{q}^{m_{q}}-t\right)$ $\subseteq C^{q}$. Let $m_{c}$ be the greatest common divisor of $\left\{m_{1}, \ldots, m_{q}\right\}$. Then $S_{t} \approx T_{t} \times R$ for $t \neq 0$ where

$$
\begin{aligned}
T_{t} & =\left\{\left(y_{1}, \ldots, y_{q}\right) \in S_{t}:\left|y_{1}\right|^{m_{1}}=\cdots=\left|y_{q}\right|^{m_{q}}\right\}, \\
R & =\left\{\left(y_{1}, \ldots, y_{q}\right) \in S_{1}: y_{i} \text { real, positive for all } i\right\} .
\end{aligned}
$$

LEMMA 3.1. For $t \neq 0, T_{t}$ consists of $m_{c}$ disjoint tori, each of real dimension $(q-1)$.

Proof. $\left(y_{1}^{m_{1}} \cdots y_{q}^{m_{q}}\right)-t$ factors into

$$
\pi\left\{y_{1}^{n_{1}} \cdots \cdots y_{q^{q}}^{n_{2}}-t_{k}: 1 \leqq k \leqq m_{c}\right\}
$$

where the $t_{k}$ are the distinct $m_{c}$-roots of $t$ and $n_{i}=\left(m_{i} / m_{c}\right) . T_{t}$ has at least one component corresponding to each factor. But for the case $m_{c}=1$, it is easily seen that $T_{t}$ is pathwise connected. Hence in general $T_{t}$ has exactly $m_{c}$ components. An induction on $q$ then gives that each factor is a $(q-1)$-torus.

( $\left.{ }^{1}\right)$ See definition in [2, Appendix A]. 
It is immediate that $R \approx\left(\boldsymbol{R}^{+}\right)^{q-1}$. Let

$$
\begin{gathered}
R_{J}=\left\{\left(r_{1}, \ldots, r_{q}\right) \in R: r_{i}^{m_{i}}=r_{j}^{m_{j}} \text { for all } i, j \in J, r_{i}^{m_{i}} \leqq r_{j}^{m_{1}} \text { for } i \in J, j \notin J\right\}, \\
R_{J, \theta}=\left\{\left(y_{1}, \ldots, y_{q}\right): \exists\left(r_{1}, \ldots, r_{q}\right) \in R_{J}, \theta_{i} \geqq 0 \text { for all } i \in J\right. \text { such that } \\
\left.\sum\left\{m_{i} \theta_{i}: i \in J\right\}=\theta, y_{i}=\left(\exp \theta_{i}\right) r_{i} \text { for } i \in J, y_{i}=r_{i} \text { for } i \notin J\right\} .
\end{gathered}
$$

Define $R_{\theta}=\bigcup\left\{R_{J, \theta}: J \subseteq\{1, \ldots, q\}, J \neq \varnothing\right\}$. Then $R_{\theta} \subseteq S_{t}$ where $t=\exp \theta$, and $R_{\theta}$ is a continuous perturbation of $R=R_{0}$.

Further, for $J \subseteq\{1, \ldots, q\}$, and a fixed constant $K>1$, define

$$
\begin{aligned}
A(J) & =\bigcap\left\{Z\left(y_{i}\right): i \in J\right\} \subseteq C^{\infty}, \\
Y(J) & =\left\{\left(y_{1}, \ldots, y_{q}:\left|y_{i}\right|^{m_{i}}<K, i \in J\right\},\right. \\
Y^{\prime}(J) & =\left\{\left(y_{1}, \ldots, y_{q}\right) \in Y(J):\left|y_{j}\right|^{m_{j}} \geqq K, j \notin J\right\}, \\
A^{\prime}(J) & =A(J) \cap Y^{\prime}(J),
\end{aligned}
$$

and let $\mu(J): \mathrm{Cl}\left(Y^{\prime}(J)\right) \rightarrow A^{\prime}(J)$ be the natural projection produced by setting $y_{\imath}=0$ for $i \in J$.

Define

$$
\varphi: R \rightarrow Q=\left\{\left(\rho_{1}, \ldots, \rho_{q}\right) \in \boldsymbol{R}^{q}: \sum \rho_{i}=1\right\}
$$

by $\rho_{i}=\left(\log K-m_{i} \log r_{i}\right) / q \log K . \sigma_{r}=R \cap Y(\{1, \ldots, q\})$ corresponds under $\varphi$ to $\sigma_{\rho}=\left\{\rho \in Q: \rho_{i} \geqq 0\right.$ for all $\left.i\right\}$. For $J \subseteq\{1, \ldots, q\}$, define $\sigma_{\rho}(J)$ to be the subsimplex spanned by the collection of vertices of $\sigma_{\rho}$ which have 1 in the $j$ th position for some $j \in J$. Let $\bar{I}=$ the closed unit interval.

Lemma 3.2. There is a $C^{\infty}$ retraction $\psi: Q \times I \rightarrow Q$ of $Q$ onto $\sigma_{\rho}$ such that

(1) $\psi(, 0)=$ identity on $Q$,

(2) $\psi(, \alpha)=$ identity on a neighborhood of $(1 / q, \ldots, 1 / q)$ for all $\alpha \in I$,

(3) $\psi(, \alpha)\left(\sigma_{\rho}(J)\right)=\sigma_{\rho}(J)$ for all $J \subseteq\{1, \ldots, q\}, \alpha \in \bar{I}$,

(4) $\psi(, 1): \varphi\left(Y^{\prime}(J) \cap Q\right) \rightarrow \sigma_{\rho}(J)$ and this map is independent of the variables $r_{j}$ for $j \notin J$.

Proof. Use induction on $q$. (This retraction is similar to the one used by Pham $[5$, p. 338].)

Define $\rho: R \rightarrow \sigma_{\rho}$ to be the composition $\psi(, 1) \circ \varphi$. Then we can define $F(\theta): S_{1}$ $\rightarrow S_{\exp \theta}$ by

$$
F(\theta)(y)=\left(\exp \left(\rho_{1} \theta / m_{1}\right) y_{1}, \ldots, \exp \left(\rho_{q} \theta / m_{q}\right) y_{q}\right)
$$

where $\left(\rho_{1}, \ldots, \rho_{q}\right)=\rho\left(\left|y_{1}\right|, \ldots,\left|y_{q}\right|\right) . F(\theta)$ is induced by a $C^{\infty}$-normal bundle construction as in $\S 1$ and its action is fibred over $A^{\prime}(J)$ by the fibrations $\mu(J)$ defined above. Further $F(\theta)(R)$ is homotopic to $R_{\theta}$ in $S_{\exp \theta}$ in such a way that

(1) the homotopy respects the fibration $\mu(J)$ of $S_{\exp \theta} \cap Y^{\prime}(J)$;

(2) under the homotopy, $F(\theta)\left(\sigma_{r}\right)$ corresponds to

$$
\sigma_{\theta}=R_{\theta} \cap \mathrm{Cl}(Y(\{1, \ldots, q\})) .
$$


If we orient $\sigma_{r}=\sigma_{0}$ by the natural order of local coordinates $r_{1}, \ldots, r_{q-1}$, then $F(\theta)$ induces an orientation on each

$$
\sigma_{J, \theta}=R_{J, \theta} \cap \mathrm{Cl}(Y(\{1, \ldots, q\})) \text { of } \sigma_{\theta} .
$$

For $J=\{1, \ldots, q\}$, this orientation is equal to $(-1)^{q-1}$ times the orientation induced by the natural order of the local coordinates $\theta_{1}, \ldots, \theta_{q-1}$. This follows from Lemma 3.2(2) and the fact that under $F(\theta)$ we have $\left(\partial \theta_{i} / \partial r_{i}\right)<0$ at $(1 / q, \ldots, 1 / q)$.

Let $m$ be any common multiple of $\left\{m_{1}, \ldots, m_{q}\right\}$. For $J=\{1, \ldots, q\}$, we consider $\sigma_{\theta}$ as a $(q-1)$-homology chain with orientation and coefficients of its simplicial decomposition given by considering $\sigma_{\theta}$ as the image under $F(\theta)$ of the oriented simplex $\sigma_{0}$. Then $\sigma_{J, m(k-1)}$ is parametrized by

$\left\{\left(\theta_{1}, \ldots, \theta_{q-1}\right): \theta_{i} \geqq 0\right.$ for all $i, \sum\left\{m_{i} \theta_{i}: 1 \leqq i \leqq q-1\right\} \leqq m k$ and $\left.m_{i_{0}} \cdot \theta_{i_{0}} \geqq m\right\}$ for any fixed $1 \leqq i_{0} \leqq q-1$. Using this fact and direct calculation one obtains that the chain

$$
\eta_{m, q-1}=\sigma_{m(q-1)}-\left(\begin{array}{c}
q-1 \\
1
\end{array}\right) \sigma_{m(q-2)}+\left(\begin{array}{c}
q-1 \\
2
\end{array}\right) \sigma_{m(q-3)}-\cdots \pm\left(\begin{array}{l}
q-1 \\
q-1
\end{array}\right) \sigma_{m \cdot 0}
$$

is a $(q-1)$-chain parametrized by $0 \leqq m_{i} \theta_{i} \leqq m, 1 \leqq i \leqq q-1$.

Let $T$ be the $(q-1)$-torus in $T_{1}$ which has nonempty intersection with $R . T$ is a topological group with a component $T^{\prime}$ of $\left\{\left(y_{1}, \ldots, y_{q}\right) \in T: y_{q-1}=1\right\}$ as a maximal connected subgroup, and

$$
\begin{aligned}
& \left\{\left(y_{1}, \ldots, y_{q}\right) \in T: y_{1}=\cdots=y_{q-2}=1 \text { and } y_{q-1}=\exp \theta_{q-1},\right. \\
& \left.0 \leqq \theta_{q-1} \leqq\left(\text { g.c.d. }\left\{m_{1}, \ldots, m_{q-2}, m_{q}\right\} / m_{c}\right)\right\}
\end{aligned}
$$

a transverse set for $T / T^{\prime}$. Thus by our remarks on orientation above and induction on $q$ we have

FORMULAE 3.3. $\eta_{m, q-1}=(-1)^{q-1}\left(m^{q-1} m_{c} / \pi\left\{m_{i}: 1 \leqq i \leqq q\right\}\right) \cdot T$ and if $S_{1}$ is oriented by its complex structure, then as an intersection number formula:

Finally, define

$$
\sigma_{0} \cdot\left(\eta_{m, q-1}\right)=(-1)^{q(q-1) / 2}\left(m^{q-1} \cdot m_{c} / \pi m_{i}\right) .
$$

$$
T_{t}(J)=\left\{\left(y_{1}, \ldots, y_{q}\right) \in S_{t}:\left|y_{i}\right|^{m_{i}}=\left|y_{j}\right|^{m_{j}} \text { for } i, j \in J\right\} .
$$

From the considerations of Lemma 3.1 it is clear that there exists an $m(J)$-sheeted complex analytic covering space $C_{t}(J)$ of $A^{\prime}(J)$, where $m(J)=$ g.c.d. $\left\{m_{i}: i \in J\right\}$, and the following commutative diagram of fibrations:

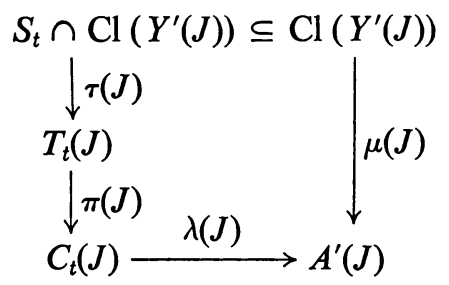


where $\pi$ has fibre a $\left(q^{\prime}-1\right)$-real torus and $\tau$ has fibre a $\left(q^{\prime}-1\right)$-simplex, $q^{\prime}=|J|$. Then for $|J|=q-1, t=1$, and $j_{0} \notin J, \lambda(J)^{-1}\left(P \in A^{\prime}(J):\left|y_{j_{0}}\right|^{m\left(j_{0}\right)}=K\right)$ has $m_{c}$ components and for $C=$ any one of these components:

(3.5) $\pi(J)^{-1}(C) \approx$ (one of the $(q-1)$-tori of $\left.T_{1}\right)$, with this isomorphism given by the projection $\tau(I): S_{1} \cap \mathrm{Cl}\left(Y^{\prime}(\{1, \ldots, q\})\right) \rightarrow T_{1}$ where $|I|=q$.

4. Vanishing bundles and the action of $F(\theta)$. We now return to the general problem considered in $\S 1$ and $\S 2$.

THEOREM 4.1. There are tubular neighborhoods $Y(i)$ of $A(i)$ in $X$ for $0 \leqq i \leqq h$ such that for $Y(I)=\bigcap\{Y(i): i \in I\}$ there is a $C^{\infty}$-normal bundle fibration

$$
\mu(I): \mathrm{Cl}(Y(I)) \rightarrow A(I) .
$$

Further the following conditions are satisfied:

(1) For $W(q)=\bigcup\{Y(I):|I|=q\}$ and $Y^{\prime}(I)=Y(I)-W(|I|+1), \quad A^{\prime}(I)=A(I)$ $-W(|I|+1)$, one has that $\mu(I)^{-1}\left(A^{\prime}(I)\right)=\mathrm{Cl}\left(Y^{\prime}(I)\right)$.

(2) For each $P \in A^{\prime}(I)$, there is a $V \in \mathscr{V}$ with $I(V)=I$ and $\mu(I)^{-1}(P) \subseteq V$, where $\mathscr{V}$ is the regular covering of Theorem 2.4.

(3) For $J \subseteq I, \mu(I) \circ \mu(J)=\mu(I)$ on $\mathrm{Cl}(Y(I))$.

(4) For each $I \subseteq\{0, \ldots, h\}$ with $|I|=q$, there is a constant $K(I)>0$ such that, for $P \in A^{\prime}(I)$ and $V \supseteq \mu^{-1}(P)$, the mapping $\mu^{-1}(P) \rightarrow C^{q}$ given by $(y(i, V)(P, Q))_{i \in I}$ is an isomorphism of $\mu^{-1}(P)$ onto the closed polycylinder with radii $K^{1 / m(i)}$ in $C^{q}$.

(5) $\mu(I)^{-1}(P) \cap A^{\prime}(J)$ is a $(|I|-|J|)$-real torus for $P \in A^{\prime}(I), J \subseteq I$.

(6) $(W(q)-\mathrm{Cl}(W(q+1)))$ is a disjoint union of open sets, one for each I with $|I|=q$.

Proof. To construct $\mu(I)$ use Theorem 2.4, parts 3(b), 4(b), and (5). Then all the properties of the theorem follow from Theorem 2.4 and the behavior of normalized coordinates as seen in $\S 3$.

Next let us construct $F(\theta): S_{1} \rightarrow S_{\exp \theta}$ which is induced by a normal bundle construction as in $\S 1$ and which behaves like the $F(\theta)$ constructed in $\S 3$ on each $\mu(I)^{-1}(P)$ for $P \in A^{\prime}(I)$. Let $\delta_{i}: X \rightarrow[0,1]$ be a $C^{\infty}$-function such that:

(1) $\delta_{i} \equiv 1$ in a neighborhood of $A(i)$;

(2) $\delta_{i} \equiv 0$ on $X-Y(i)$;

(3) for $P \in A^{\prime}(I), i \in I$, and $Q, Q^{\prime} \in \mu^{-1}(P) \subseteq V$ with

$$
|y(i, V)(P, Q)|=\left|y(i, V)\left(P, Q^{\prime}\right)\right|
$$

we have $\delta_{i}(Q)=\delta_{i}\left(Q^{\prime}\right)$. For $t$ sufficiently small, we have for each $Q \in S_{t}$ that $\delta_{i}(Q)=1$ for some $i \in\{1, \ldots, h\}$. We can assume that we have this condition for $|t|<2$. Define, for $Q \in S_{1}$,

$$
\rho_{i}(Q)=\left(\delta_{i}(Q) / \sum\left\{\delta_{j}(Q): 1 \leqq j \leqq h\right\}\right)-\delta_{0}(Q)
$$

for $1 \leqq i \leqq h$ and $\rho_{0}(Q)=\delta_{0}(Q)$. 
Define $F(\theta, P): \mu(I)^{-1}(P) \cap S_{1} \rightarrow \mu(I)^{-1}(P) \cap S_{\exp \theta}$ for $P \in A^{\prime}(I)$ by:

$$
F(\theta, P)\left((y(i, V)(P, Q))_{i \in I}\right)=\left(\left(\exp \left(\rho_{i}(Q) \theta / m(i)\right) y(i, V)(P, Q)\right)_{i \in I}\right)
$$

where $V \supseteq \mu(I)^{-1}(P)$. Theorem 2.4, parts (3) and (4), shows that this definition is independent of the choice of $V$ and that the $F(\theta, P)$ piece together to give a $C^{\infty}$ mapping:

$$
F(\theta): S_{1} \rightarrow S_{\exp \theta} .
$$

This mapping is easily seen to be induced by a normal bundle construction as described in $\S 1$. Hence we can assume that, whenever $\left\{S_{t}\right\}$ acquires an ordinary singularity at $t=0$, then $F(\theta)$ is as constructed in (4.2).

We now proceed to introduce the other constructions used in $\$ 3$ to the general case with the help of normalized coordinates and the fibrations $\mu(I)$.

On $\mu(I)^{-1}(P)$ with $P \in A^{\prime}(I)$, we will let $\left(y_{i}\right)_{i \in I}$ be any of the coordinate systems $(y(i, V)(P,))_{i \in I}$ for $V \supseteq \mu^{-1}(P)$ and $I(V)=I$. By Theorem 2.4, the choice of $V$ will not matter in what follows.

For $0 \notin I$, define

$$
T_{t}(I)=\left\{Q \in S_{t} \cap Y^{\prime}(I):\left|y_{i}\right|^{m(i)}=\left|y_{j}\right|^{m(j)} \text { for } i, j \in I\right\} .
$$

Then for $t$ sufficiently small, hence we assume for $0<|t|<2, T_{t}(I) \cap \mu(I)^{-1}(P)$ is, by Lemma 3.1, $m(I)$ real tori each of real dimension $(|I|-1)$, for $P \in A^{\prime}(I) .(m(I)$ $=$ greatest common divisor of $\{m(i): i \in I\}$.) Then there exists a complex manifold $C_{t}(I)$, which is an $m(I)$-sheeted covering space of $A^{\prime}(I)$ and such that $T_{t}(I)$ is a $(q-1)$-torus bundle over $C_{t}(I)$, where $q=|I|$.

Further we can define a fibration $\tau(I):\left(S_{t} \cap \mathrm{Cl}\left(Y^{\prime}(I)\right)\right) \rightarrow T_{t}(I)$ by

$$
\tau(I)^{-1}(Q)=\left\{Q^{\prime} \in\left(S_{t} \cap \mu(I)^{-1}(P)\right): \operatorname{Arg} y_{i}\left(Q^{\prime}\right)=\operatorname{Arg} y_{i}(Q) \text { for all } i \in I\right\}
$$

where $P=\mu(I)(Q)$. Then we have a commutative diagram of projections for $0<|t|<2$ :

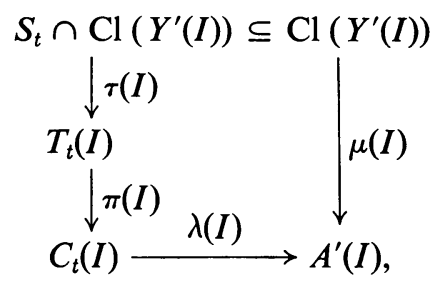

which is a replica of (3.4) on each $\mu(I)^{-1}(P), P \in A^{\prime}(I)$.

By (3.5) and Theorem 4.1(5), $\pi(I): T_{1}(I) \rightarrow C_{1}(I)$ can be factored into a series of circle bundles. Hence the composition of the Gysin maps associated to each of the circle bundles gives maps:

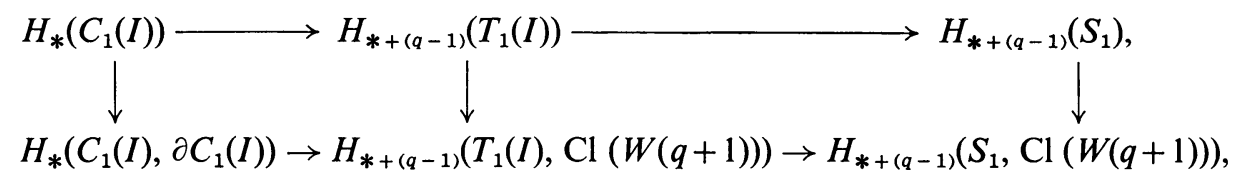


where, intuitively, the horizontal maps take a cycle of $C_{1}(I)$ into the fibre above it in $T_{1}(I)$. Since it will cause no confusion, we will denote both of the horizontal composition maps by $\psi(I)$.

By $\S 3, \tau(I)$ has fibre a $(q-1)$-simplex so that we can apply the Thom-Gysin isomorphism and construct:

$$
H_{*}\left(S_{1}\right) \rightarrow H_{*}\left(S_{1}, K(I)\right) \approx H_{*-(q-1)}\left(T_{1}(I), \mathrm{Cl}(W(q+1))\right)
$$

where $K(I)=\mathrm{Cl}\left(S_{1}-Y^{\prime}(I)\right)$. Call this composition $\chi(I)$.

Now let $m=$ a common multiple of $\{m(1), \ldots, m(h)\}$. Define $\eta_{m}=F(m)_{*}$-(identity) : $H_{*}\left(S_{1}\right) \rightarrow H_{*}\left(S_{1}\right)$ where $F(\theta)_{*}$ is as in $\S 1$ and hence can be assumed to be defined by $F(\theta)$ in (4.2). Since there is little danger of confusion, we shall use $\eta_{m}$ also to refer to the analogous

$$
F(m)_{*} \text {-(identity): } H_{*}\left(S_{1}\right) \rightarrow H_{*}\left(S_{1}, \mathrm{Cl}(W(q+1))\right) .
$$

We are now ready to state and prove a generalized Poincaré formula.

Let $\Gamma_{p}(I)$ denote a basis for $H_{p}\left(C_{1}(I), \partial C_{1}(I)\right)$, and let $\Gamma_{p^{\prime}}(I)$ denote its dual basis in $H_{p^{\prime}}\left(C_{1}(I)\right)$ where $p+p^{\prime}=2(n-q+1)$. Assume $C_{1}(I)$ to be oriented from the complex structure of $A^{\prime}(I)$. For $\gamma \in \Gamma_{p}(I)$ we shall denote its dual by $\gamma^{\prime}$. Finally let $\mathscr{J}_{q}=\{I \subseteq\{1, \ldots, h\}:|I|=q\}$.

THEOREM 4.4. The mapping $\left(\eta_{m}\right)^{q-1}: H_{p+q-1}\left(S_{1}\right) \rightarrow H_{p+q-1}\left(S_{1}, \mathrm{Cl}(W(q+1))\right)$ is given by

$$
\left(\eta_{m}\right)^{q-1}(\tilde{\alpha})=(-1)^{r} m^{q-1} \sum\left\{\left(m(I) / \pi_{I} m(i)\right)\left(\tilde{\alpha} \cdot \psi\left(\gamma^{\prime}\right)\right) \psi(\gamma): \gamma \in \Gamma_{p}(I), I \in \mathscr{J}_{q}\right\}
$$

where $r=(q(q-1) / 2)+p(q-1)$.

Proof. For $0 \in I$, let $T_{1}(I)=C_{1}(I)=A^{\prime}(I)$ and define $\tau(I)=\left.\mu(I)\right|_{s_{1} \cap \mathrm{Cl}\left(Y^{\prime}(l)\right)}$ $\pi(I)=\lambda(I)=$ identity map. We will need a series of lemmas.

Lemma 4.5. For $0 \in I, P \in A(I), \mu(I)^{-1}(P) \cap S_{1}$ is an analytic polydisc of complex dimension $|I|-1$.

Proof of lemma. By Definition 2.1, $A(I)$ is a nonsingular subvariety of $S_{1}$. Further $\mu(I): Y(I) \rightarrow A(I)$ is a normal bundle in $X$. Hence each $\mu(I)^{-1}(P)$ must intersect $S_{1}$ normally.

LEMMA 4.6. There exists a finite cellular decomposition of $B(1)=S_{0} \cup S_{\infty}$ such that:

(1) The decomposition respects the chain of subspaces $B(1) \supseteq B(2) \supseteq \cdots B(n+2)$ $=\varnothing$ as well as each of the subspaces $A^{\prime}(I), I \subseteq\{0, \ldots, h\}$;

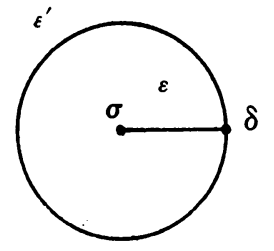


(2) for a p-cell $\sigma$ of $A^{\prime}(I)$, there is a $V \in \mathscr{V}$ (see Theorem 2.4) with $\mu(I)^{-1}(\sigma) \subseteq V$ and $I=I(V)$; and for $\varepsilon=\left\{Q \in\left(A(J) \cap \mathrm{Cl}\left(Y^{\prime}(I)\right): y_{j}(Q)\right.\right.$ real $\left.\geqq 0\right\}$ and

$$
\varepsilon^{\prime}=\left\{Q \in\left(A(J) \cap \mathrm{Cl}\left(Y^{\prime}(I)\right):\left|y_{j}(Q)\right|=K(I)^{1 / m(j)}\right\}\right.
$$

and $\delta=\varepsilon \cap \varepsilon^{\prime}$ with $J \cup\{j\}=I$, we have $\varepsilon, \varepsilon^{\prime} \subseteq(p+1)$-skeleton of $A(J), \delta \subseteq p$-skeleton of $A^{\prime}(J)$;

(3) the decomposition lifts through $\lambda$ to a cellular decomposition of each $C_{1}(I)$.

Proof of lemma. (1) and (3) are immediate from the elementary theory of $C-W$ complexes. For (2) use Theorem 4.1(5).

Define

$$
Y_{k}=\bigcup\left\{\pi(I)^{-1}\left(\pi(I)^{-1}\left(p \text {-skeleton of } C_{1}(I)\right)\right): I \subseteq\{0, \ldots, h\}, p+|I|=k\right\} .
$$

LEMMA 4.7. The natural mapping $H_{k-1}\left(Y_{k}\right) \rightarrow H_{k-1}\left(S_{1}\right)$ is onto.

Proof of lemma. Let $K_{g}=\left(S_{1}-W(g)\right) \cup Y_{k}$ and assume $H_{k-1}\left(K_{g+1}\right) \rightarrow H_{k-1}\left(S_{1}\right)$ is onto. We have an exact sequence:

$$
H_{k-1}\left(K_{g}\right) \rightarrow H_{k-1}\left(K_{g+1}\right) \rightarrow H_{k-1}\left(K_{g+1}, K_{g}\right) .
$$

However by Lemma 4.6(2), and using the Thom-Gysin isomorphism for $\tau(I)$ (see (4.3) and Lemma 4.5), we have for $p=k-g$ and $T_{1}(I)_{p}=\pi(I)^{-1}(p$-skeleton of $\left.C_{1}(I)\right)$ :

$$
H_{k-1}\left(K_{g+1}, K_{g}\right) \approx \sum\left\{H_{p}\left(T_{1}(I), T_{1}(I)_{p}: I \subseteq\{0, \ldots, h\},|I|=g\right\} .\right.
$$

Using that $\tau(I)$ can be factored into circle bundles, each with an ordinary Gysin sequence, it is easily concluded that:

$$
H_{p}\left(T_{1}(I), T_{1}(I)_{p}\right) \approx H_{p}\left(C_{1}(I), p \text {-skeleton }\right)=0 .
$$

Hence $H_{k-1}\left(K_{g}\right)$ is onto by the composition of two surjective maps.

LEMMA 4.8. The natural mapping $H_{k-1}\left(Y_{k}\right) \rightarrow H_{k-1}\left(Y_{k}, Y_{k-1}\right)$ is injective.

Proof of lemma. It suffices to show that $H_{k-1}\left(Y_{k-1}\right)=0$. Define

$$
L_{g}=\bigcup\left\{\tau(I)^{-1}\left(\pi(I)^{-1}\left(p \text {-skeleton of } C_{1}(I)\right)\right): p+|I|=k-1 \text { and }|I| \geqq g\right\} \text {. }
$$

Suppose $H_{k-1}\left(L_{g+1}\right)=0$. $L_{g}$ has as a strong deformation retract the space

$$
L_{g+1} \cup \bigcup\left\{T_{1}(I)_{p}:|I|=g\right\}
$$

for $p=k-1-g$. However each $T_{1}(I)$ has dimension at most $p+(|I|-1)=k-2$. Hence $H_{k-1}\left(L_{g}, L_{g+1}\right)=0$ and therefore $H_{k-1}\left(L_{g}\right)=0$.

$F(m)$ as constructed in (4.2) respects all the subspaces constructed above. Hence by Lemmas 4.7 and 4.8 , it suffices to characterize

$$
\eta_{m}=F(m)_{*} \text {-(identity): } H_{k-1}\left(Y_{k}, Y_{k-1}\right) \rightarrow H_{k-1}\left(Y_{k}, Y_{k-1}\right) \text {. }
$$

Let $\sigma$ be a $p$-cell of $C_{1}(I)$ for $p+|I|=k$. Let $M=\tau(I)^{-1}\left(\pi(I)^{-1}(\sigma)\right)$. Then the boundary of $M$, which we shall denote by $M^{*}$, is equal to the union of 
$N=\tau(I)^{-1}\left(\pi(I)^{-1}\left(\sigma^{*}\right)\right)$ and $\tilde{G}=M-W(|I|)$. Finally let $G=\tilde{G} \cap Y_{k-1}$. Then $H_{k-1}\left(Y_{k}, Y_{k-1}\right)$ is the direct sum of groups of the form:

$$
H_{k-1}(M, G \cup N) \text {; }
$$

hence it suffices to consider $\eta_{m}: H_{k-1}(M, G \cup N) \rightarrow H_{k-1}(M, G \cup N)$, since each subspace of this type is preserved by $F(m)$ as constructed in (4.2). Further we have the exact sequence:

$$
H_{k-1}(\tilde{G}, G) \rightarrow H_{k-1}(M, G \cup N) \rightarrow H_{k-1}(M, \tilde{G} \cup N) .
$$

Using Lemma 4.6(2), the generator for $H_{k-1}(M, \tilde{G} \cup N)$ can be represented by the cell

$$
\omega=\left\{Q \in M: y_{i}(Q)=r_{i} \bar{y}_{i} \text { for all } i \in I \text { for some }\left(r_{j}\right) \in R\right\}
$$

where $R$ is as in $\S 3$ and $\bar{y}_{i}=y_{i}(\bar{Q})$ for any fixed $\bar{Q} \in \pi(I)^{-1}(\sigma)$. For the proper choice of $\bar{Q}, \omega^{\circ} \in G \cup N$ by the construction of the cellular decomposition in Lemma 4.6(2).

(4.12) Since $H_{k-1}(\tilde{G}, G)$ is itself a direct sum of groups of the form (4.9), but over $p$-cells $\sigma^{\prime}$ of $C_{1}\left(I^{\prime}\right)$ with $p^{\prime}+|I|=k$ and $\left|I^{\prime}\right|<|I|$ we can inductively assume that any element of $H_{k-1}(\widetilde{G}, G)$ has a representative $\omega^{\prime}$ which is a sum of cells of type (4.11) with real coefficients over $p^{\prime}$-cells $\sigma^{\prime}$ with $p^{\prime}>p$.

By (4.10), for any cycle $\alpha$ of $H_{k-1}(M, G \cup N)$, there is a representative chain of the form $a(\sigma) \omega+\omega^{\prime}$ with $\omega$ and $\omega^{\prime}$ as in (4.11) and (4.12) and $a(\sigma) \in \boldsymbol{R}$. Now by (4.2) and the first of Formulae 3.3, $(F(m)-\text { (identity) })^{q-2}\left(\omega^{\prime}\right)$ is homologous to a sum of chains of the form $\pi\left(I^{\prime}\right)^{-1}\left(\sigma^{\prime}\right)$ where $\sigma^{\prime}$ is as in (4.12) and $q=|I|$. But $F(m)$ as constructed in (4.2) is homotopic in each fibre to the identity on each $\pi\left(I^{\prime}\right)^{-1}\left(\sigma^{\prime}\right)$. Hence $(F(m)-(\text { identity }))^{q-1}\left(\omega^{\prime}\right)$ is homologous to 0 in $H_{k-1}(M, G \cup N)$. Further by the same formula we have that up to sign:

(4.13) $(F(m)-\text { (identity) })^{q-1}(\omega) \sim\left(m^{q-1} m(I) / \pi_{I} m(i)\right) T_{\sigma}$ if $I \in \mathscr{J}_{q}$, where

$$
T_{\sigma}=\left\{Q \in M:\left|y_{i}(Q)\right|^{m(i)}=\left|y_{j}(Q)\right|^{m(j)} \text { for all } i, j \in I\right\},
$$

and $(F(m)-(\text { identity }))^{q-1}(\omega) \sim 0$ otherwise. $(\sim$ indicates homologous to in $H_{k-1}(M, G \cup N)$.)

Now let $\tilde{\alpha} \in H_{p+q-1}\left(S_{1}\right)$. Then $\tilde{\alpha}$ has a representative cycle $\alpha$ in $Y_{p+q}$ which is made up of cells of the form $a(\sigma) \omega$ with $\omega$ as in (4.11). Further, for $I \in \mathscr{J}_{q}$,

$$
\sum\left\{a(\sigma) \sigma: \sigma p \text {-cell of } C_{1}(I)\right\}
$$

is, with appropriate orientation, the cycle of $H_{p}\left(C_{1}(I), \mathrm{Cl}(W(q+1))\right)$ given by $\pi(I)(\chi(I)(\tilde{\alpha}))$. $(\chi(I)$ was defined earlier in $\S 4$.) The theorem then follows from (4.13) and the fact that orientation for the mapping $\chi(I)$ can be chosen so that:

$$
\left(\left(\tilde{\alpha} \cdot\left(\psi(I)\left(\gamma^{\prime}\right)\right)\right) \text { in } S_{1}\right)=(-1)^{p(q-1)}\left(\left((\pi(I)(\chi(I)(\tilde{\alpha}))) \cdot \gamma^{\prime}\right)\right.
$$

in $\left.C_{1}(I)\right)$. The calculation of the sign in the theorem is then essentially given by the second formula of Formulae 3.3. This completes the proof of Theorem 4.4. 
Note 4.14. Let $H_{0}(I)$ be the bundle associated to $\lambda \circ \pi: T_{1}(I) \rightarrow A^{\prime}(I)$ with fibre $H_{0}\left(\left(\lambda_{0} \pi\right)^{-1}(P)\right)$ for $P \in A^{\prime}(I)$, (see [7, p. 151]). Then

$$
\begin{aligned}
H_{p}\left(C_{1}(I)\right) & \approx H_{p}\left(A^{\prime}(I) ; H_{0}(I)\right), \\
H_{p}\left(C_{1}(I), \partial C_{1}(I)\right) & \approx H_{p}\left(A^{\prime}(I), \partial A^{\prime}(I) ; H_{0}(I)\right) .
\end{aligned}
$$

Corollary 4.15. Let $\alpha$ be an element of $H_{k-1}\left(S_{1}-W(q+1)\right)$. Then

$$
\eta_{m}^{q-1}(\alpha)=(-1)^{r} m^{q-1} \sum\left\{\left(m(I) / \pi_{I} m(i)\right)(\alpha \cdot \psi(\gamma)) \psi\left(\gamma^{\prime}\right): \gamma^{\prime} \in \Gamma_{p}^{\prime}(I), I \in \mathscr{J}_{q}\right\}
$$

in $H_{k-1}\left(S_{1}\right)$ where $r=(q(q-1) / 2)+p q$.

Proof. Choose a representative cycle for $\alpha$ in $\left(Y_{k}-W(q+1)\right)$ and proceed as in Theorem 4.4. Note that $\left(\gamma^{\prime} \cdot \gamma\right)=(-1)^{p}$.

In the case $q=1$, Theorem 4.4 is true if we define $\eta_{m}^{0}$ to be the standard homology map

$$
H_{*}\left(S_{1}\right) \rightarrow H_{*}\left(S_{1}, \mathrm{Cl}(W(2))\right) \text {. }
$$

However this provides no new information. For the sake of completeness, we shall add here the more appropriate result for the case $q=1$. For $I \in \mathscr{J}_{1}$ and

$$
M(I)=\{\exp (k / m(I)): k \in Z\}
$$

with generator $\xi(I)=\exp (1 / m(I))$, we have that $M(I)$ acts on $S_{1} \cap \mathrm{Cl}\left(Y^{\prime}(I)\right)$ as in (4.2). For $\gamma \in H_{*}\left(C_{1}(I), \partial C_{1}(I)\right), \psi(I)(\gamma)=\gamma$. Let

$$
\tilde{\psi}(I)(\gamma)=(\xi(I) \cdot \gamma)-\gamma
$$

As in [5], the group-ring $Z(M(I))$ acts on $H_{*}\left(C_{1}(I), \partial C_{1}(I)\right)$. Define $\tilde{k}=\exp (k / m(I))$.

THEOREM 4.16. For $\alpha \in H_{p}\left(S_{1}\right)$ and $b \geqq 1$, then, in $H_{p}\left(S_{1}, \mathrm{Cl}(W(2))\right)$,

$$
\eta_{b}(\alpha)=\sum\left\{\left(\alpha \cdot \gamma^{\prime}\right) \tilde{k} \cdot \Psi(\gamma): 0 \leqq k \leqq b-1, \gamma \in \Gamma_{p}(I), I \in \mathscr{J}_{1}\right\} .
$$

In particular, if $b$ is a multiple of $m(i)$ for all $1 \leqq i \leqq h$, then $\eta_{b}(\alpha)=0$ in $H_{p}\left(S_{1}, \mathrm{Cl}(W(2))\right)$.

Proof. Immediate from the fact that $\eta_{b}=F(b)_{*}$-(identity) and from the construction of $F(\theta)$ in (4.2). Note that, for

$$
\xi=\sum\{\tilde{k}: 1 \leqq k \leqq m(I)\} \in Z(M(I))
$$

we have $\xi \cdot \psi(\gamma)=0$ for all $\gamma \in \Gamma_{p}(I)$.

Corollary 4.17 Let $\alpha$ be an element of $H_{p}\left(S_{1}-\mathrm{Cl}(W(2))\right)$. Then

$$
\eta_{b}(\alpha)=(-1)^{p} \sum\left\{(\alpha \cdot \gamma) \tilde{k} \cdot \tilde{\psi}\left(\gamma^{\prime}\right): 0 \leqq k \leqq b-1, \gamma^{\prime} \in \Gamma_{p}^{\prime}(I), I \in \mathscr{J}_{1}\right\}
$$

in $H_{p}\left(S_{1}\right)$. 
5. Applications. The proof of Theorem 4.4 actually shows the action of $F(l)_{*}: H_{*}\left(S_{1}\right) \rightarrow H_{*}\left(S_{1}\right)$ for any $l \geqq 0$. For if $\alpha \in H_{k-1}\left(S_{1}\right)$, then there is a $\beta \in H_{k-1}\left(Y_{k}, Y_{k-1}\right)$ which has a common preimage with $\alpha$ in $H_{k-1}\left(Y_{k}\right)$. Further, $\beta$ has as representative a sum of cells of the form $a_{\omega} \cdot \omega$ with $a_{\omega} \in \boldsymbol{R}$ and $\omega$ as in (4.11). Then $F(l)_{*}(\beta)$ is represented in $H_{k-1}\left(Y_{k}, Y_{k-1}\right)$ by the sum of cells $a_{\omega} \cdot \omega_{l}$ where

$$
\omega_{l}=\left\{Q \in M: y_{i}(Q)=r_{i} \bar{y}_{i} \text { for all } i \in I \text { for some }\left(r_{j}\right) \in R_{l}\right\}
$$

with $M$ as in (4.9) and $R_{l}$ as in $\S 3$. By Lemma 4.8, such an element of $H_{k-1}\left(Y_{k}, Y_{k-1}\right)$ comes from a unique element of $H_{k-1}\left(Y_{k}\right)$ which in turn determines $F(l)_{*}(\alpha)$ $\in H_{k-1}\left(S_{1}\right)$. However, in general this is an unwieldy process.

In this final section we will give a formula which helps characterize $F(m)_{*}: H_{*}\left(S_{1}\right)$ $\rightarrow H_{*}\left(S_{1}\right)$ without necessitating the explicit construction outlined above. ( $m$ here, as before, is a common multiple of $m(i)$ for $1 \leqq i \leqq h$.) With this formula and Theorem 4.4 we will completely construct

$$
\eta_{m}=F(m)_{*}-\text { (identity): } H_{*}\left(S_{1}\right) \rightarrow H_{*}\left(S_{1}\right)
$$

for the case $n=2$. (The case $n=0$ is trivial and for $n=1$, which corresponds to the classical Picard-Lefschetz formula, $\eta_{m}$ is completely given by Theorem 4.4.)

FORMULA 5.1. Let $\alpha$ be an element of $H_{p^{\prime}+q-1}\left(S_{1}-W(q+2)\right)$. Then for all $\beta \in H_{p+q-1}\left(S_{1}\right)$ with $p+p^{\prime}=2(n-q)$ :

$$
\begin{aligned}
\left(\eta_{m}^{q-1}(\alpha) \cdot \beta\right) & +(-1)^{q}\left(\alpha \cdot \eta_{m}^{q-1}(\beta)\right) \\
= & (-1)^{r}(q-1) m^{q} \sum\left\{\left(m(I) / \pi_{I} m(i)\right)(\alpha \cdot \psi(\gamma))\left(\psi\left(\gamma^{\prime}\right) \cdot \beta\right): \gamma \in \Gamma_{p}(I), I \in \mathscr{J}_{q+1}\right\}
\end{aligned}
$$

where $r=((q+1) q / 2)+p(q+1)$.

Proof. By Corollary 4.15, the right-hand side of the formula is simply $(q-1) \eta_{m}^{q}(\alpha) \cdot \beta$. However $\eta_{m}^{q}(\alpha)$ is invariant under $F(m)_{*}$ and $F(m)$ is an orientationconserving automorphism. Hence $\eta_{m}^{q}(\alpha) \cdot \beta=\eta_{m}^{q}(\alpha) \cdot F(m)_{*}^{k}(\beta)$ for all $k \in Z$. Thus the right-hand side of the formula is equal to

$$
\sum\left\{\eta_{m}^{q}(\alpha) \cdot F(m)_{*}^{k}(\beta): 1 \leqq k \leqq q-1\right\}
$$

which in turn is equal to:

$$
\sum\left\{(-1)^{(q-j)}\left(\begin{array}{l}
q \\
j
\end{array}\right) F(m)_{*}^{j}(\alpha) \cdot F(m)_{*}^{k}(\beta): 0 \leqq j \leqq q, 1 \leqq k \leqq q-1\right\}
$$

This last summation reduces to $\eta_{m}^{q-1}(\alpha) \cdot \beta+(-1)^{q}\left(\alpha \cdot \eta_{m}^{q-1}(\beta)\right)$ by repeated use of the equality:

$$
F(m)_{*}^{j}(\alpha) \cdot F(m)_{*}^{k}(\beta)=F(m)_{*}^{j+i}(\alpha) \cdot F(m)_{*}^{k+i}(\beta) \text { for all } i \in \boldsymbol{Z} .
$$

Now let us examine $\eta_{m}: H_{j}\left(S_{1}\right) \rightarrow H_{j}\left(S_{1}\right)$ for $n=2$. For $j=0$ and $4, \eta_{m} \equiv 0$ trivially. For $j=1, \eta_{m}$ is given by Corollary 4.15 . For $j=3, \eta_{m}$ is given by Theorem 4.4 since the natural map $H_{3}\left(S_{1}\right) \rightarrow H_{3}\left(S_{1}, \mathrm{Cl}(W(3))\right)$ is an injection. We shall now 
characterize $\eta_{m}: H_{2}\left(S_{1}\right) \rightarrow H_{2}\left(S_{1}\right)$. Note that $W(4)=\varnothing$ and so for any $\alpha, \beta \in H_{2}\left(S_{1}\right)$ we have that:

$$
\eta_{m}(\alpha) \cdot \beta+\alpha \cdot \eta_{m}(\beta)=(-1) m^{2} \sum\left\{\left(m(I) / \pi_{I} m(i)\right)(\alpha \cdot \psi(\gamma))(\beta \cdot \psi(\gamma)): \gamma \in \Gamma_{0}(I), I \in \mathscr{J}_{3}\right\}
$$

by Formula 5.1. Define the right-hand side of this expression to be equal to $\Phi(\alpha, \beta)$. This formula has as corollaries that:

(5.2) For all $\alpha, \beta \in H_{2}\left(S_{1}\right): \eta_{m}(\alpha) \cdot \eta_{m}(\beta)=-\Phi(\alpha, \beta)$ since $\alpha \cdot \eta_{m}^{2}(\beta)=\Phi(\alpha, \beta)$ by Theorem 4.4;

(5.3) $\eta_{m}^{2}(\alpha)=0$ for $\alpha \in H_{2}\left(S_{1}\right)$ if and only if $\eta_{m}(\alpha) \cdot \eta_{m}(\alpha)=0$ (since $\eta_{m}(\alpha) \cdot \eta_{m}(\alpha)=0$ implies $(\alpha \cdot \psi(\gamma))=0$ for $\left.\gamma \in \Gamma_{0}(I), I \in \mathscr{J}_{3}\right)$.

Let

$$
E=\bigcup\left\{A(I): I \in \mathscr{J}_{3}\right\}
$$

( $E$ is called the set of triple points of the hypersurface $S_{0}$.) Let $B=\lambda^{-1}(E)$ and for each $P \in C(I), I \in \mathscr{J}_{3}$, let $T(P)=\pi(I)^{-1}(P), W(P)=\tau(I)^{-1}\left(\pi(I)^{-1}(P)\right)$. Let $\bar{Q}_{P}$ be fixed in $T(P)$ and let $\omega_{P}$ be as in (4.11). Let

$$
S_{1}^{\prime}=\left(S_{1}-W(3)\right) \cup \bigcup\left\{\omega_{P}: P \in B\right\} .
$$

Then by the Thom-Gysin isomorphism and Lemma 4.5, $H_{2}\left(S_{1}^{\prime}\right) \rightarrow H_{2}\left(S_{1}\right)$ is onto. Let

and let

$$
D(J)=\pi(J)\left(\bigcup\left\{T_{1}(J) \cap \omega_{P}: P \in B\right\}\right)
$$

$$
K=\bigcup\left\{\tau(J)^{-1}\left(\pi(J)^{-1}(Q): Q \in D(J)\right\} .\right.
$$

Then, as in $\S 4$, we define for $J \in \mathscr{J}_{2}$ :

$$
\begin{aligned}
& \psi(J): H_{1}\left(C_{1}(J), D(J)\right) \rightarrow H_{2}\left(S_{1}-W(3), K\right) \\
& \tilde{\chi}(J): H_{2}\left(S_{1}^{\prime}\right) \rightarrow H_{1}\left(C_{1}(J), D(J)\right)
\end{aligned}
$$

with orientations chosen as in the final paragraph of the proof of Theorem 4.4. $(\tilde{\chi}(J)$ corresponds to $\pi(J) \circ \chi(J)$ of $\S 4$.)

For each $P \in B$, let $\tau_{P}$ be a generator of $H_{2}(W(P))=H_{2}(T(P))$. Let $\left\{\tau_{1}, \ldots, \tau_{s}\right\}$ be a maximal linearly independent set in $\left\{\tau_{P}: P \in B\right\}$ and let $\left\{\alpha_{1}, \ldots, \alpha_{s}\right\}$ be a dual set under the intersection pairing in $H_{2}\left(S_{1}\right)$.

LEMMA 5.4. There are unique elements $\eta_{i} \in H_{2}\left(S_{1}\right)$ for $1 \leqq i \leqq s$ such that there exist elements $\xi_{P} \in H_{2}(W(P), K)$ for $P \in B$ such that:

(1) if $\varphi_{i}=-m \sum\left\{\left(m(J) / \pi_{J} m(j)\right) \psi(J) \circ \tilde{\chi}(J)\left(\alpha_{1}^{\prime}\right): J \in \mathscr{J}_{2}\right\}$ where $\alpha_{i}^{\prime}$ is some preimage of $\alpha_{i}$ in $H_{2}\left(S_{1}^{\prime}\right)$, then the image of $\eta_{i}$ in $H_{2}\left(S_{1}, K\right)$ is given by

$$
\varphi_{i}+\sum\left\{\left(\alpha_{i} \tau_{P}\right) \xi_{P}: P \in B\right\}
$$

(2) for $1 \leqq i, j \leqq s, \eta_{i} \alpha_{j}+\alpha_{i} \eta_{j}=\Phi\left(\alpha_{i}, \alpha_{j}\right)$.

Proof. There is a representative of $\alpha_{i}^{\prime}$ of the form

$$
\varepsilon+\sum\left\{\left(\alpha_{i} \cdot \tau_{P}\right) a_{P} \omega_{P}: P \in B\right\}
$$


where $\varepsilon$ is a cycle of $\left(S_{1}-W(3)\right)$ relative to $K$ and the $a_{P}$ are constants in $\boldsymbol{R}$. Then $F(m)$ fixes $\left(S_{1}-W(3)\right), K$, and $W(P)$ for each $P$ by (4.2), and so:

(1) $\eta_{m}: H_{2}\left(S_{1}-W(3), K\right) \rightarrow H_{2}\left(S_{1}-W(3), K\right)$ takes $\{\varepsilon\}$ into $\varphi_{i}$ by the proof of Theorem 4.4;

(2) $\eta_{m}: H_{2}(W(P), K) \rightarrow H_{2}(W(P), K)$ takes $\left\{a_{P} \omega_{P}\right\}$ into an element of $H_{2}(W(P), K)$ which we will call $\xi_{P}$.

Then putting $\eta_{i}=\eta_{m}\left(\alpha_{i}\right)$, the existence of a collection $\left\{\eta_{i}\right\}$ satisfying the lemma is proved. For uniqueness, suppose $\eta_{i}, \eta_{i}^{\prime}$ for $1 \leqq i \leqq s$ satisfy the conditions of the lemma. Let $\xi_{P}, \xi_{P}^{\prime}$ be the corresponding elements of $H_{2}(W(P), K)$ for all $P \in B$. Then

$$
\eta_{i}-\eta_{i}^{\prime}=\sum\left\{\left(\alpha \cdot \tau_{P}\right)\left(\xi_{P}-\xi_{P}^{\prime}\right): P \in B\right\}
$$

in $H_{2}\left(S_{1}, K\right)$. However $H_{2}(K)=0$ and $\partial_{*}\left(\xi_{P}-\xi_{P}^{\prime}\right)=0$ in $H_{1}(K)$ and so $\left(\xi_{P}-\xi_{P}^{\prime}\right)$ comes from an element $b_{P} \tau_{P}$ in $H_{2}\left(S_{1}\right)$. To get the uniqueness of the $\eta_{i}$ it suffices to show that for $1 \leqq i \leqq s$ :

$$
\sum\left\{\left(\alpha_{i} \cdot \tau_{P}\right) b_{P} \tau_{P}: P \in B\right\}=0 .
$$

But by condition (2) of the lemma:

$$
\left(\eta_{i}-\eta_{i}^{\prime}\right) \cdot \alpha_{j}+\alpha_{i} \cdot\left(\eta_{j}-\eta_{j}^{\prime}\right)=0 .
$$

Hence for all $1 \leqq j \leqq s$, we have that:

$$
2 \sum\left\{b_{P}\left(\alpha_{i} \cdot \tau_{P}\right)\left(\alpha_{j} \cdot \tau_{P}\right): P \in B\right\}=0 .
$$

Since the $\alpha_{j}$ form a dual basis to the subspace of $H_{2}\left(S_{1}\right)$ generated by $\left\{\tau_{P}: P \in B\right\}$, this suffices to prove $(*)$.

CoROllaRY 5.5. $\eta_{i}=\eta_{m}\left(\alpha_{i}\right) \in H_{2}\left(S_{1}\right)$ for all $1 \leqq i \leqq s$.

Now $\Gamma_{1}(J)$, the basis for $H_{1}\left(C_{1}(J), \partial C_{1}(J)\right)$ for $J \in \mathscr{J}_{2}$, can be so chosen that:

$$
\Gamma_{1}(J)=\Delta_{1}(J) \cup \Omega_{1}(J), \quad \Gamma_{1}^{\prime}(J)=\Delta_{1}^{\prime}(J) \cup \Omega_{1}^{\prime}(J)
$$

where $\Delta_{1}(J)$ generates the image of $H_{1}\left(C_{1}(J)\right)$ in $H_{1}\left(C_{1}(J), \partial C_{1}(J)\right)$ and $\Omega_{1}^{\prime}(J)$ generates the image of the mapping $H_{1}\left(\partial C_{1}(J)\right) \rightarrow H_{1}\left(C_{1}(J)\right)$ and $\gamma \in \Delta_{1}(J)$ implies that $\gamma^{\prime} \in \Delta_{1}^{\prime}(J)$. For any $\alpha \in H_{2}\left(S_{1}\right)$,

$$
\alpha^{\prime \prime}=\alpha-\sum\left\{\left(\alpha \cdot \tau_{i}\right) \alpha_{i}: 1 \leqq i \leqq s\right\}
$$

comes from an element (which we shall also call $\left.\alpha^{\prime \prime}\right)$ of $H_{2}\left(S_{1}-W(3)\right)$. By Corollary 4.15,

$$
\eta_{m}\left(\alpha^{\prime \prime}\right)=(-1) m \sum\left\{\left(m(J) / \pi_{J} m(j)\right)\left(\alpha^{\prime \prime} \cdot \psi(\gamma)\right) \psi\left(\gamma^{\prime}\right): \gamma^{\prime} \in \Gamma_{1}^{\prime}(J), J \in \mathscr{J}_{2}\right\}
$$

in $H_{2}\left(S_{1}\right)$. Now $\Gamma_{1}^{\prime}(J)=\Delta_{1}^{\prime}(J) \cup \Omega_{1}^{\prime}(J)$ and for $\gamma^{\prime} \in \Omega_{1}^{\prime}(J), \psi\left(\gamma^{\prime}\right)$ is contained in the subspace of $H_{2}\left(S_{1}\right)$ generated by $\left\{\tau_{P}: P \in B\right\}$ by (3.5). Hence

$$
\begin{aligned}
\eta_{m}\left(\alpha^{\prime \prime}\right)= & (-1) m \sum\left\{\left(m(J) / \pi_{J} m(j)\right)\left(\alpha^{\prime \prime} \cdot \psi(\gamma)\right) \psi\left(\gamma^{\prime}\right): \gamma \in \Delta_{1}(J), J \in \mathscr{J}_{2}\right\} \\
& +\sum\left\{b_{i}\left(\alpha^{\prime \prime}\right) \tau_{i}: 1 \leqq i \leqq s\right\} .
\end{aligned}
$$


However by Formula 5.1 and Corollary 5.5, $\left(\alpha^{\prime \prime} \cdot \eta_{i}\right)+\left(\eta_{m}\left(\alpha^{\prime \prime}\right) \cdot \alpha_{i}\right)=\Phi\left(\alpha^{\prime \prime}, \alpha_{i}\right)=0$ for all $1 \leqq i \leqq s$. Thus intersecting this last formula for $\eta_{m}\left(\alpha^{\prime \prime}\right)$ with $\alpha_{i}$ we have that:

$$
b_{i}\left(\alpha^{\prime \prime}\right)=m \sum\left\{\left(m(J) / \pi_{J} m(j)\right)\left(\alpha^{\prime \prime} \cdot \psi(\gamma)\right)\left(\alpha_{i} \cdot \psi\left(\gamma^{\prime}\right)\right): \gamma \in \Delta_{1}(J), J \in \mathscr{J}_{2}\right\}-\left(\alpha^{\prime \prime} \cdot \eta_{i}\right) .
$$

Define

$$
\tilde{\psi}(\gamma)=\psi(\gamma)-\sum\left\{\left(\alpha_{i} \cdot \psi(\gamma)\right) \tau_{i}: 1 \leqq i \leqq s\right\} \in H_{2}\left(S_{1}\right)
$$

for $\gamma \in \Delta_{1}(J), J \in \mathscr{J}_{2}$. Then $\alpha \cdot \tilde{\psi}(\gamma)=\alpha^{\prime \prime} \cdot \psi(\gamma)$. Define

$$
\begin{aligned}
\tilde{\eta}_{i}= & \eta_{i}-m \sum\left\{\left(m(J) / \pi_{J} m(j)\right)\left(\alpha_{i} \cdot \psi\left(\gamma^{\prime}\right)\right) \tilde{\psi}(\gamma): \gamma \in \Delta_{1}(J), J \in \mathscr{J}_{2}\right\} \\
& -\sum\left\{\left(\alpha_{k} \cdot \eta_{i}\right) \tau_{k}: 1 \leqq k \leqq s\right\}
\end{aligned}
$$

THEOREM 5.6 For $\alpha \in H_{2}\left(S_{1}\right)$, then

$$
\begin{aligned}
\eta_{m}(\alpha)= & \sum\left\{\left(\alpha \cdot \tau_{i}\right) \eta_{i}: 1 \leqq i \leqq s\right\} \\
& -m \sum\left\{\left(m(J) / \pi_{J} m(j)\right)(\alpha \cdot \tilde{\psi}(\gamma)) \psi\left(\gamma^{\prime}\right): \gamma \in \Delta_{1}(J), J \in \mathscr{J}_{2}\right\} \\
& -\sum\left\{\left(\alpha \cdot \tilde{\eta}_{i}\right) \tau_{i}: 1 \leqq i \leqq s\right\}
\end{aligned}
$$

in $H_{2}\left(S_{1}\right)$, where $m$ is a common multiple of $m(j)$ for all $1 \leqq j \leqq h$.

Proof. The formula is an immediate consequence of the formulae and definitions just preceding.

I would like to thank Professor Phillip A. Griffiths of the University of California at Berkeley for suggesting the problem which led to this paper and for many valuable conversations.

\section{BIBLIOGRAPHY}

1. C. Clemens, Picard-Lefschetz theorem for families of algebraic varieties acquiring certain singularities, Doctoral Dissertation, Berkeley, Calif., 1966 (unpublished)( ${ }^{2}$ ).

2. R. Gunning and H. Rossi, Analytic functions of several complex variables, Prentice-Hall, Englewood Cliffs, N. J., 1965.

3. S. Lefschetz, L'analysis situs et la géométrie algébrique, Gauthier-Villars, Paris, 1924.

4. J. Milnor, Differentiable structures, Mimeographed notes, Princeton Univ., Princeton, N. J., 1961.

5. F. Pham, Formules de Picard-Lefschetz généralisées et ramification des intégrales, Bull. Soc. Math. France 93 (1965), 333-367.

6. E. Picard and G. Simart, Théorie des fonctions algébrique de deux variables indépendantes. I, Gauthier-Villars, Paris, 1897; Chapitre IV.

7. N. Steenrod, The topology of fibre bundles, Princeton Univ. Press, Princeton, N. J., 1951.

Universidad Técnica del Estado,

Santiago de Chile

$\left(^{2}\right)$ (In the above reference, the author's treatment of the triple point case is incorrect.) 